\title{
The Information Needs of School-based Speech- Language Pathologist Assistants: A Case Study ${ }^{1}$
}

\author{
Shea Matthew Betts
}

\begin{abstract}
Speech-language pathologists (SLPs) are professionals who specialize in diagnosing and treating communication disorders. They are sometimes supported by a speech-language pathologist assistant (SLPA), who engages in treatment procedures under the guidance of the supervising SLP. Both the qualifications needed to practice as well as the scope of responsibilities vary for SLPAs depending on jurisdiction. Notably, these assistants can play a central role in the treatment of speech disorders. Research regarding the information needs of SLPAs, however, is limited. This paper seeks to explore the information resources and services available to a particular SLPA community and to examine the obstacles to meeting its information needs. An interview with a practicing school-based SLPA is used to discuss current practices and suggest improvements to information services available to SLPA communities. This interview highlights some of the challenges that may be faced by school-based SLPAs when seeking information, and it provides an opportunity to consider context-specific solutions to these issues.
\end{abstract}

\section{Introduction}

Speech-language pathologists (SLPs) are professionals who diagnose and treat communication disorders. They may be assisted by paraprofessionals who perform tasks related to the treatment of communication disorders under the guidance of their supervising SLP. The training and certification necessary to be a speech-language pathologist assistant (SLPA) varies geographically. Speech-Language and Audiology Canada [1] recognizes a variety of diplomas and certificates for SLPAs, which consist of both formal training and fieldwork. Although the scope and settings of an SLPA's practice varies based on provincial guidelines, they may be responsible for preparing programming materials, assisting with formal and informal documentation, participating in family conferences with a supervising SLP, using screening protocols, and assisting the SLP with patients as needed [1]. SLPAs may also be responsible for assisting with departmental operations, research projects, in-service training, and both family and community education [1]. The manner by which SLPAs access the information needed to perform these duties offers a unique area of investigation in the field of information studies. This paper aims to instigate a discussion regarding information access and information literacy in a schoolbased SLPA community.

\section{Literature discussion}

Although the information needs of SLPs have been examined, research in this area is limited [2-6]. Kloda and Bartlett [2] acknowledged that further research is required to gain a better understanding of the information behaviour of rehabilitation therapists. It is important to consider how SLPs access and use information when examining the needs of SLPAs, especially considering their shared objectives of therapy. Considering that few studies examine the information needs of SLPs, the lack of scholarly literature regarding the same needs of their assistants is perhaps not surprising. In addition to similarities between SLP and SLPA information needs, parallels with SLPA information needs can be drawn from research focusing on the information needs of other medical assistants and interdisciplinary professionals, especially in the areas of occupational therapy, physical therapy, and social work.

According to Kloda and Bartlett [2], knowledge about the clinical information behaviour of rehabilitation therapists, consisting of occupational therapists, physical therapists, and SLPs, is limited. Reviewing 17 studies dating from 1990, only four of which concerned SLPs [3-6], a study by Kloda and Bartlett [2] examined the information needs, information seeking, and information use of rehabilitation therapists. The authors noted that the

Shea Matthew Betts ${ }^{2}$, Master of Information Studies, McGill University, Montréal, QC

${ }^{1}$ This article was the recipient of the 2015 JCHLA / JABSC Student Paper Prize. This article has been peer reviewed.

${ }^{2}$ Corresponding author (e-mail: shea.m.betts@gmail.com) 
information needs of rehabilitation therapists stem largely from the context of patient care [2]. The review also found that rehabilitation therapists favour journal articles, books, their colleagues, as well as nonprofessional websites as sources of information, rather than databases [2]. Another study by Harrison et al. [7] found similar preferences among social workers in the United Kingdom, who favour personal verbal communication, directories, and procedure manuals for meeting their information needs. In terms of obstacles to meeting information needs, one study observed that rehabilitation therapists identify a lack of skills and time limitations as impediments to finding information [2]. Despite any connections that may be drawn between SLPs and SLPAs, it is important to recognize that the information behaviour of a therapy assistant may be very different from that of a rehabilitation therapist.

Although there is little information regarding the information needs and uses of rehabilitation therapists, there is even less research that looks specifically at the information needs, uses, and services available to therapy assistants. To gain a better sense of these, it is necessary to examine medical assistants and interdisciplinary professionals. One study by Bertulis and Cheeseborough [8] examined the information needs of nurses, health care assistants, and other medical staff in the United Kingdom with the aim of developing information services using evidence-based practice. Despite the need to access information through the internet for work-related inquiries, the researchers found that a substantial amount of health workers do not have internet access when required [8]. A lack of internet access was also found for social workers in the United Kingdom, preventing easy access to current research [7]. Health workers surveyed by Bertulis and Cheeseborough [8] identified five potential improvements for information services: improved access to computers and the internet, improved information literacy training, more time to study or research, improved access to full-text journals, and the provision of a list of useful websites for the employee's specialization. The authors concluded that health workers require a blended service comprised of physical resources, support from colleagues, supervisors, and medical librarians, as well as online information [8]. The importance of online information, including an efficient internet infrastructure, for social workers to implement evidence-based practice is echoed in other studies [7]. When examining information needs in the medical and social work sectors, the significance of internet access to current research must not be overlooked.

The ability to assess resources is an important factor when considering how to deliver effective therapy. A study by Nail-Chiwetalu and Bernstein Ratner [4] that examined the information seeking abilities of practicing SLPs found that that a majority of respondents used search engines and databases of scholarly research interchangeably to access clinical information. Given the variation of currency and reliability of sources found on the internet, either through search engines or databases, the information literacy skills of practicing therapists is of significant interest to researchers. Notably, the same study found that few SLPs had received librarian-led information literacy training [4]. The authors suggested that information literacy training should be incorporated into the education of SLP graduate students, as well as through learning opportunities for practicing therapists [4]. Other research recognized the importance of information literacy for social workers and suggests that the appointment of an information specialist can be a powerful force when moving towards evidence-based practice [7]. Although knowledge regarding information literacy opportunities for SLPAs is lacking, such research highlights the value of information literacy instruction to those working in the field of speech-language therapy and other interdisciplinary areas.

Other learning opportunities available to SLPAs are an important consideration, especially those that enhance the interpersonal information seeking practices of assistants. Paul-Brown and Goldberg [9] observed that providing regular meetings in which different treatment approaches are discussed could be utilized as learning opportunities for SLPAs. Similarly, discussion groups have been proposed as a way to update social workers with the latest information [7]. Establishing online communication networks could also enhance how SLPAs meet their information needs, allowing the exchange of ideas between assistants, SLPs, and even the classroom teachers whose students are being treated [9]. How such networks could be organized, implemented, and used provides an interesting area of future research. Meetings and communication networks can serve as a means of allowing SLPAs to access expertise and provides a way in which practical treatment information can be transferred between assistants.

\section{Methods}

In the fall of 2014, an SLPA working in a large city in Alberta, Canada, was interviewed via Skype to gain an understanding of her information needs and uses. Such an interview can provide an understanding of a user's information behaviour, defined by Wilson [10] as the actions involving the identification of one's information needs, the search process for the information, and the use or transfer of such information. Owing to the exploratory nature of the study, only one SLPA, with over 10 years of experience in the field, was interviewed. Identifying as fairly typical in comparison with her colleagues, the SLPA is employed by a provincial health care organization, and, although she is based in an office, she works primarily in elementary school settings, travelling between several schools. The patients undergoing treatment are elementary public school students. The SLPA, along with several colleagues based at the same office, reports to a supervising SLP. In addition to her information needs, SLPA responsibilities, training, learning opportunities, and obstacles to meeting her information needs were also discussed in a semi-structured interview to obtain insight into the information needs and information behaviour of the SLPA community. 


\section{Results}

The interview provided an illuminating glimpse into the information needs and behaviours of school-based SLPAs. The participant received six weeks of training after being hired, which consisted of formal training and fieldwork. She expressed that professional development opportunities to help meet SLPA information needs are limited, observing that there have been few in the past five years. No information literacy programs have been provided to her either during her training or since she has begun her work, and she has no access to librarians specializing in speechlanguage pathology. She identified monthly, optional, SLP-led videoconferences that discuss practical techniques that can be used during treatment as the most useful learning opportunity offered.

The SLPA views her supervising SLP as her primary source of treatment information, her colleagues as her secondary source, followed by websites found through internet search engines. Frequent communication between the SLP, the assistant, and her colleagues is conducted by phone, email, written notes, and in person when possible. Print resources are available at the health organization office regarding speech and language disorders, which are chosen and purchased by an SLP leader. Resources are reproduced and brought out to treatment sessions, and can be used for patient homework. Print resources are available for only the most common speech problems, which the participant identified as a potential impediment to treatment.

SPLAs frequently seek information in the context of their work, and the participant identified three major areas of information needs: patient, behaviour management, and treatment information. Patient information involves demographic information, such as language, that can guide treatment. Behaviour information is required when a patient's behaviour hinders treatment. The SLPA identified treatment information as the most important need. Although prescribed treatment methods are provided by the SLP, issues can arise that require the assistant to seek out information.

The SLPA identified two main obstacles to meeting her information needs. First, different locations of resources and treatment make it difficult to treat a child. If a patient is found to have additional speech difficulties, treatment for those will have to wait until the next treatment session, when the SLPA can bring all the appropriate resources. Another challenge involves uncommon speech difficulties, and a lack of related resources. It is in this situation that the participant seeks information from outside of her colleagues and SLP, primarily from the use of internet search engines.

\section{Discussion}

The SLPA interviewed acknowledged three areas of information needs including patient, behaviour management, and treatment information. The participant had no difficulties accessing patient or behaviour information, which is conducted through discussions with a patient's parents and teacher, respectively. Treatment information, however, was identified by the participant as the most important area of her information needs, as well as where she faced the most significant barriers. Although she finds some services conducive to meeting her information needs, such as SLP-led teleconferences that discuss practical treatment techniques, as well as print materials for most speech issues, a lack of other services and resources make meeting these needs difficult. Unfortunately, a number of obstacles exist for the participant when attempting to meet her information needs.

A collection of print materials is available for SLPAs to use during treatment, but only for the most common speech disorders. These include word lists to be used during treatment as well as physical copies of exercises that patients can work on at home. For SLPAs, who are not trained to develop these word lists, a lack of materials for uncommon speech issues presents a challenge. Notably, the SLPA interviewed received no training about how to find information during training or since she began her work. Despite this, she is sometimes required to evaluate information found through search engines regarding topics that are not covered by the print materials available to her. Although the importance of information literacy training for SLPs has been recognized [4], similar sessions could provide an opportunity for SLPAs to gain a better understanding of how to search for and evaluate resources. For example, some of the monthly teleconferences could be devoted to information literacy. Another option would be to incorporate information literacy sessions into professional development opportunities as often as deemed necessary by supervisors. These sessions would need to be applicable to SLPAs, targeting their particular information needs, and could be led by a specialized librarian appointed to the speech therapy office or an experienced SLPA. Although the SLPA can check if a word list is usable with the SLP, basic searching and evaluating skills could increase the likelihood of the SLPA finding an acceptable source. If certain print resources, especially word lists, are not made available to SLPAs, their ability to select appropriate and reliable alternative resources might play an important role in patient treatment.

Another service that could be provided to SLPAs, either as a substitute for or compliment to information literacy sessions, is the creation of a resource list of approved resources. Such a list, which could be made available to SLPAs either as a physical copy or accessible online, should outline suitable websites that could be used for practical treatment purposes, including word lists provided by such sites. Experienced SLPs should be responsible for approving such a directory. Much like information literacy sessions, resource lists have the potential for improving the efficiency of treatment. Instead of SLPAs using search engines to locate a potentially unreliable word list for an uncommon speech problem and then obtaining approval from their SLP, they could refer to a directory with preapproved resources.

The SLPA interviewed is based in an office, where print materials for treatment are located. Treatment, however, occurs in multiple schools, and SLPAs must commute between the office and these schools, selecting and copying whatever materials are necessary for treatment from the 
office. If a patient is found to have an additional speech issue, they must wait until the following session for the SLPA to bring the appropriate resource. With a limited number of therapy sessions per year, ensuring comprehensive treatment during each session is paramount. To improve the efficiency of resource access for SLPAs, online access to treatment resources could be provided, consisting of the aforementioned resource list, as well as digitized versions of the print resources found at their office. This could be as simple as a website with relevant links and PDF files, or more sophisticated in the form of a database. Although the creation and maintenance of such an online resource would require a financial commitment by management, and would take time to develop, it would allow SLPAs to have access to the required treatment materials in the field. Studies have emphasized the importance of internet access for social workers and paraprofessional medical staff $[7,8]$, which may have implications in other fields such as speech-language pathology. By eliminating the distance barriers between resources and place of treatment, SLPAs can maximize their treatment time with patients if additional resources are needed for treatment.

How SLPAs access these online resources might present a challenge. The SLPA interviewed indicated a desire to use either a laptop or iPad in the field, primarily when searching for uncommon speech disorder word lists, but also when a patient is found to have additional difficulties with certain sounds. The participant also noted that while SLPAs working in nearby districts have these tools available to them, she would need to rely on inconsistent access to whatever computer is available at a given school. Although supplying SLPAs with devices to access electronic resources may be costly, management must weigh this with the costs of providing and replacing incomplete physical resources.

\section{Conclusion}

The information needs of the SLPA interviewed illustrate the challenges of accessing information in a paraprofessional context. Although she can often depend on her colleagues and supervising SLP for information, complications occasionally arise that require her to become a more self-directed information seeker. A variety of improvements to training and services, including information literacy sessions, a collection of approved internet resources, communication networks, and tools to access the internet while working at treatment sites may contribute to a solution. It is important to note that this study draws from an interview with a single individual whose perspectives are not necessarily shared by her colleagues.
The inferences and suggestions outlined in this paper are intended to serve as a catalyst to facilitate further discussion about the information needs of a seldomstudied community. Systematic investigations into what information SLPAs require, as well as how they access and use it, may provide an opportunity to improve information services for this unique community.

\section{References}

1. Speech-Language and Audiology Canada. Speech-language pathology assistant guidelines. [Internet]. Ottawa, ON: Speech-Language and Audiology Canada; c2015. 2004 [updated 2014 Oct; cited 2014 Nov 20]. Available from: http:// sacoac.ca/sites/default/files/resources/CHA_Guidelines_SLPAssistant_EN.pdf.

2. Kloda LA, Bartlett JC. Clinical information behavior of rehabilitation therapists: a review of the research on occupational therapists, physical therapists, and speech-language pathologists. J Med Libr Assoc. 2009;97(3):194-202. PMID: 19626145. doi: 10.3163/1536-5050.97.3.008.

3. Guo R, Bain BA, Willer J. Results of an assessment of information needs among speech-language pathologists and audiologists in Idaho. J Med Libr Assoc. 2008;96(2):138-44. PMID: 18379669. doi: 10.3163/1536-5050.96.2.138.

4. Nail-Chiwetalu B, Bernstein Ratner N. An assessment of the information-seeking abilities and needs of practicing speechlanguage pathologists. $J$ Med Libr Assoc. 2007;95(2):182-8. PMID: 17443251. doi: 10.3163/1536-5050.95.2.182.

5. Haigh V. Clinical effectiveness and allied health professionals: an information needs assessment. Health Info Libr J. 2006; 23(1):41-50. PMID: 16466498. doi: 10.1111/j.1471-1842. 2006.00635.x.

6. Vallino-Napoli LD, Reilly S. Evidence-based health care: a survey of speech pathology practice. Adv Speech Lang Pathol. 2004;6(2):107-12. doi: 10.1080/14417040410001708530.

7. Harrison J, Hepworth M, de Chazal P. NHS and social care interface: a study of social workers' library and information needs. J Libr Info Sci. 2004;36(1):27-36. doi: 10.1177/0961000604042971.

8. Bertulis R, Cheeseborough J. The Royal College of Nursing's information needs survey of nurses and health professionals. Health Info Libr J. 2008;25(3):186-97. PMID: 18796079. doi: 10.1111/j.1471-1842.2007.00755.x.

9. Paul-Brown D, Goldberg LR. Current policies and new directions for speech-language pathology assistants. Lang Speech Hear Serv Sch. 2001;32(1):4-17. doi: 10.1044/01611461(2001/001).

10. Wilson TD. Models in information behaviour research. $J$ Doc. 1999;55(3):249-70. doi: 10.1108/EUM0000000007145. 